\title{
Use of the Nottingham Health Profile with patients after a stroke
}

\author{
SHAH EBRAHIM, ${ }^{1 *}$ DAVID BARER, ${ }^{2}$ AND FIONA NOURI ${ }^{2}$ \\ From the Department of Health Care of the Elderly, ${ }^{1}$ Queens Medical Centre, Nottingham University Medical \\ School, Nottingham NG7 2UH; and the Stroke Research Unit Offices, ${ }^{2}$ General Hospital, Nottingham
}

SUMmaRY The Nottingham Health Profile (NHP) is easy to use with stroke patients and may be used with those who cannot manage more complicated mood questionnaires, such as the General Health Questionnaire (GHQ). Stroke patients rate their health, and especially emotions and feelings of social isolation, as much worse than that of people of similar age. NHP emotion scores correlate with objective measures of disability, length of hospital stay, and GHQ scores. The NHP is a valid indicator of depressed mood, and combining its components into a total score gives the greatest accuracy in detecting depression. Patients with high scores at one month continued to report large numbers of problems at six months after their stroke. Many patients experienced pain, disturbed sleep, and social isolation, which are important, potentially treatable problems not usually considered in the management of stroke patients. Many patients with problems did not see their general practitioner or any other source of help, and additional follow up was needed.

The Nottingham Health Profile (NHP) is a questionnaire designed to measure social and personal effects of illness. It is used as a measure of need for health care and as an outcome measure in evaluation. Among groups of subjects it correlates well with objective measures of health status and is sensitive to changes in disease severity. ${ }^{1-4}$ It has 38 questions (requiring a yes/no response) on energy, pain, emotion, sleep, social isolation, and mobility, and the scores on each component are weighted to give a score from 0 to $100 .^{5}$ It can be completed in about 5 minutes.

The aim of the study was to describe the social and psychological problems experienced by patients after a stroke and the extent to which NHP scores were associated with physical disability, use of services, length of hospital stay, and an affective illness inventory, the General Health Questionnaire (GHQ). ${ }^{6}$

\section{Methods}

Over eight months 463 patients with acute stroke were admitted to Nottingham hospitals. Two hundred and thirty five $(51 \%)$ survived to six months and all but 13 were followed up ( 8 had moved and 5

*Present address: Department of Clinical Epidemiology and Social Medicine, S George's Hospital Medical School, Cranmer Terrace, Tooting, London SW17 ORE. refused follow up). One hundred and fifty nine (72\%) and $198(89 \%)$ had NHP assessments at one and six months respectively, and 153 were assessed on both occasions. Dysphasia and mental impairment were the main reasons for missed assessments (at one month, dysphasia 26, mental impairment 9, administrative error 20 , moved out of Nottingham area 8 ; at six months, dysphasia 15, mental impairment 7, administrative error 2). Mobility questions of the NHP were not asked at six months since detailed enquiry about physical mobility was made as part of the measurement of disability. The questions were inappropriate for some of the more handicapped hospital patients, which led to misunderstandings, and it was thought that their omission would be unlikely seriously to bias reporting of other problems. Physical disability of all patients was measured using a ranked activities of daily living (ADL) scale ${ }^{7}$ at one and six months, and 153 patients also had a GHQ assessment at six months.

A control group was selected randomly from the age-sex register of a large group practice. All patients were sent the NHP for completion and return by post. The response rate was $90 \%$ and, of the remainder, who were all visited by their family doctor, only two had important physical illness.

All analysis was done using non-parametric statistical tests (Mann-Whitney U, Kruskal-Wallis 
ANOVA) but the tables give mean NHP scores to aid interpretation of significant differences.

\section{Results}

Comparisons with age-matched control group and with disability

Table 1 compares the mean scores of each component of the NHP at one and six months for the stroke patients and the control group. NHP scores were correlated with the severity of physical disability at both one and six months, and on average scores were about twice as high among patients still unable to walk (table 2). NHP scores were also associated with place of residence (home or hospital) and length of hospital stay but not with age, sex, and living alone. One month pain, emotion, isolation, and mobility scores were high among those patients whose ability deteriorated or improved over the subsequent five months, whereas patients whose ability remained static had the lowest NHP scores (table 3).

Relation between one and six month NHP scores Weak correlations between one and six month sleep

Table 1 Mean NHP scores at one and six months after a stroke compared with an age-matched control group

\begin{tabular}{|c|c|c|c|}
\hline \multirow[b]{3}{*}{ NHP problem } & \multicolumn{3}{|c|}{ Mean age (yr) } \\
\hline & \multirow{2}{*}{ 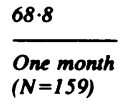 } & \multirow{2}{*}{$\begin{array}{l}69.5 \\
\text { Six months } \\
(N=198)\end{array}$} & \multirow{2}{*}{$\begin{array}{l}71 \cdot 8 \\
\text { Controls } \\
(N=200)\end{array}$} \\
\hline & & & \\
\hline Energy & $35 * *$ & $40^{* *}$ & 19 \\
\hline Pain & $11^{*}$ & $13^{*}$ & 9 \\
\hline Emotion & $21^{* *}$ & $25^{* *}$ & 9 \\
\hline Sleep & 22 & 26 & 21 \\
\hline Isolation & $20^{* *}$ & $23 * *$ & 6 \\
\hline Mobility & $21 * *$ & -+ & 14 \\
\hline
\end{tabular}

Significance tests:

* p $<0.0001$. $\mathrm{p}<0.01$.

+ Mobility questions were not asked at six months post stroke.

Table 2 Mean NHP scores at one and six months depending on level of physical ability

\begin{tabular}{|c|c|c|c|c|}
\hline \multirow[b]{3}{*}{ NHP problem } & \multicolumn{4}{|c|}{ Level of ability } \\
\hline & \multicolumn{2}{|l|}{ One month } & \multicolumn{2}{|l|}{ Six months } \\
\hline & $\begin{array}{l}\text { Unable to } \\
\text { walk } \\
(N=57)\end{array}$ & $\begin{array}{l}\text { Able to } \\
\text { walk } \\
(N=102)\end{array}$ & $\begin{array}{l}\text { Unable to } \\
\text { walk } \\
(N=33)\end{array}$ & $\begin{array}{l}\text { Able to } \\
\text { walk } \\
(N=165)\end{array}$ \\
\hline Energy & 42 & 31 & 54 & $37^{*}$ \\
\hline Pain & 15 & $7^{* *}$ & 24 & $11^{* *}$ \\
\hline Emotion & 30 & $16^{* * *}$ & 38 & $22^{* *}$ \\
\hline Sleep & 31 & $17^{* *}$ & 25 & 27 \\
\hline Isolation & 28 & $15^{* *}$ & 38 & $20^{* * * * *}$ \\
\hline Mobility & 31 & $16^{*}$ & - & - \\
\hline
\end{tabular}

Table 3 Change in physical (ADL) ability (one to six months) and mean NHP score at one month

\begin{tabular}{llll}
\hline & \multicolumn{3}{l}{ Change in physical ability } \\
\cline { 2 - 4 } $\begin{array}{l}\text { NHP problem } \\
\text { at one month }\end{array}$ & $\begin{array}{l}\text { Worse } \\
(N=18)\end{array}$ & $\begin{array}{l}\text { No change } \\
(N=65)\end{array}$ & $\begin{array}{l}\text { Better } \\
(N=76)\end{array}$ \\
\hline Energy & 39 & 33 & 36 \\
Pain & 16 & 8 & $11^{*}$ \\
Emotion & 22 & 16 & $25^{*}$ \\
Sleep & 16 & 21 & 24 \\
Isolation & 28 & 15 & $22^{*}$ \\
Mobility & 45 & 15 & $20^{* *}$ \\
\hline
\end{tabular}

* $p<0.05$. * $p<0.01$.

and energy scores were found (Spearman's $\rho 0 \cdot 25$ and 0.36 respectively), but pain, emotion, and isolation scores were more strongly correlated (Spearman's $\rho 0.55,0.53$, and 0.53 respectively). Patients with high NHP scores at one month were likely to have high scores at six months.

\section{Use of services and NHP scores}

Use of rehabilitation and medical outpatient services at six months were not related to six month NHP scores but, not surprisingly, socially isolated patients were more likely to get 'meals on wheels' and support from a home help. District nurses were much more likely to see patients with problems in any NHP area, but general practitioners (GPs) saw only patients who complained of pain more often than expected. Fifty three $(\mathbf{2 7 \%})$ patients with apparent problems had not seen or received any health or social services contact in the preceding month.

\section{Does the NHP measure depression?}

There was a strong correlation between NHP emotion questions and GHQ at six months (Spearman's $\rho \mathbf{0} \cdot 71$ ). A GHQ score of 12 or more out of 28 is usually taken as a criterion of important mood disturbance (virtually all depression) among patients with neurological disability. ${ }^{8}$ Therefore the accuracy of the NHP as an indicator of depression was measured using a GHQ cut-off of 12 or more and different thresholds for the emotion component alone and for the five component scores together. The most accurate discrimination between the depressed (GHQ score 12+) and others was obtained by using the total NHP score (summing energy, pain, emotion, sleep, and isolation scores and dividing by 5) at a threshold of 30 or more out of 100 . Table 4 shows that the sensitivity and specificity were $91 \%(31 / 34)$ and $82 \%(97 / 119)$ respectively. The nine emotion questions alone were almost as sensitive $(86 \%)$ but produced more false positives (sensitivity $=73 \%$ ) when the same threshold was used. 
Table 4 Accuracy of the NHP in detection of patients with high $G H Q$ scores

\begin{tabular}{lcc}
\hline \multirow{2}{*}{ Total NHP score } & \multicolumn{2}{c}{ General Health Questionnaire score } \\
\cline { 2 - 3 } & $12+$ & $0-11$ \\
\hline $30+$ & 31 & 22 \\
$0-29$ & 3 & 97 \\
Totals & 34 & 119 \\
\hline
\end{tabular}

\section{Discussion}

The NHP has been developed for a range of different uses: as an outcome measure for interventions, as a measure of the prevalence of self-perceived problems and unmet need, and as a monitor of changes in health status over time. The profile has been used with many different groups: mine rescue workers, fit and chronically ill elderly, pregnant women, patients with fractures, osteoarthritis, and peripheral vascular disease. The levels of problems found in this study of stroke patients were of similar size to those reported by chronically ill elderly but are lower than values of patients with osteoarthritis." It is possible that the patients with osteoarthritis were more physically handicapped than the present series of stroke patients and consequently reported more problems.

The profile was easy to use with stroke patients and was managed by some patients who could not complete the GHQ, which is more complicated. Part II of the profile was not used because at the time of study design only limited evaluations of repeatability and validity had been published. ${ }^{1}$

\section{Comparison with an age-matched control group}

Stroke patients had little energy and were emotionally distressed and socially isolated compared to people of similar age drawn at random from general practitioner lists. It is possible that the fact of being admitted as an acute emergency, rather than the stroke itself, provoked the problems reported. However, differences were found at one month, when half the patients were home, and persisted to six months, when only $11 \%$ were still in hospital. Additionally, the strong associations between impaired ability, mood, and NHP problems suggested that the stroke itself was an important determinant of social and psychological distress, which is supported by other workers. ${ }^{910}$

\section{Associations with NHP scores}

There were strong associations between some NHP problems and physical disability. One month NHP scores were related to change in ability; in particular, poor mobility, isolation, pain, and emotional distress at one month were all significantly associated with deterioration of physical ability by six months. Paradoxically, patients who improved also had higher scores than those whose ability did not change. Changing ability might be a more potent cause of self-perceived health problems than a static status. Consequently, high NHP scores at one month cannot be used as predictors of likely deterioration in ability.

Average scores did not change over the study period despite improvement in physical ability, which implied that the NHP was measuring dimensions of illness experience that were independent of objective indicators of functional ability. Intervention studies in rehabilitation should not rely only on an ADL assessment of ability as an outcome measure but should include the NHP (or similar 'quality of life' indicator) since improved physical ability may not be coupled with improved self-perceived health.

Among those patients who did get worse, depressed mood and pain may have been contributing factors. Pain was usually associated with arthritis of hips, knees or spine. Symptoms were probably aggravated by the hemiparesis causing the patient to put more weight on the unaffected side. Analgesics have a place in improving symptoms, and it is possible that relieving pain might improve spasticity and function.

Surprisingly, age, sex, and living alone had little influence on NHP scores, but other studies of factors associated with well-being have not found age or sex effects once associated physical illness is allowed for. ${ }^{11} 12$ The NHP does show a strong relation with age when younger people are also considered, ${ }^{13}$ but in this study the majority of patients were elderly and consequently age was not a determinant of NHP scores.

\section{Use of services}

Patients suffering pain were more likely to see their GP, but other self-perceived problems did not result in extra consultations. District nurses visited patients with high NHP scores more often, perhaps because they were better able to respond to the social and psychological consequences of stroke that the GP might consider to be 'non-medical'. However, over a quarter of patients with problems were not receiving help from the GP, district nurse, hospital or social services, which implied that routine follow up systems used by hospitals and community services were not sensitive to the needs of stroke patients.

\section{Detecting depression}

Patients with high emotion scores at one month did not improve, suggesting that these patients were not suffering merely from an acute reaction to their illness. Comparisons of the nine NHP emotion questions with the GHQ show that they are of value 
in picking up patients with depression and that false-positive rates can be reduced by combining the NHP components to derive an overall score. It is possible that weighting component scores (perhaps by a ranking of severity, duration or intrinsic 'nastiness' of each type of symptom) might give a more accurate discrimination between patients with and without depression. However, despite the simplicity of adding up component scores and dividing by the number of components included, an overall accuracy of $84 \%$ (see table 4) was obtained. Further work relating NHP scores to psychiatric assessments of mood might achieve greater discrimination than this simple approach.

Undoubtedly patients with high NHP scores should be questioned more closely about possible depressive illness as they may benefit from anti-depressant treatment ${ }^{14}$ or require more support from family or social and rehabilitation workers. Perhaps most significantly, some relatives were very surprised by the patient's NHP responses and asked for a copy of the NHP to give to the family doctor. Several patients (not otherwise emotionally labile) burst into tears when asked some of the more emotionally charged questions since this was one of the few opportunities they had to express their extreme distress. The NHP gives an opportunity to ask questions that patients and relatives think are meaningful.

The NHP does have disadvantages, some of which have been discussed. ${ }^{4}$ Firstly, summary presentation of results is not allowed for as scores on up to six different components have to be considered. The present study has used a simple additive model to combine scores and has demonstrated a high level of discrimination between depressed and other patients. Although more sophisticated models might be developed to try to improve discrimination, it could be argued that the NHP is not merely a depression or disability scale, and that each component measures an independent domain that merits separate consideration. However, the NHP does not consider some areas that are of great concern to handicapped patients and health workers (for example, bladder function, stigma, memory and intellectual ability, financial difficulty) and so cannot be used as a single, comprehensive assessment. Secondly, perceived health is assessed by the presence of symptoms, thus zero scores do not necessarily indicate a sense of well-being. Lastly, thresholds for 'abnormal' responses have not been set as previous validations have compared NHP scores of groups with differing ability or status, rather than examining the accuracy of the profile in classifying individuals according to some more objective 'gold standard' of disability or mood. Alternatively, thresholds might be set above which individuals would have a high probability of benefiting from intervention.

The present study has attempted to provide thresholds to identify patients with depression, has highlighted the problems of emotional distress, unalleviated pain, and social isolation, and has demonstrated the role of the NHP in auditing the care received by stroke patients.

SE was supported by a Wellcome Trust Clinical Epidemiology Training fellowship, DB by an ICI Pharmaceuticals Ltd stroke research fellowship, and FN by the Nottingham Stroke Appeal Fund.

\section{References}

${ }^{1}$ Hunt SM, McKenna SP, McEwan J, et al. A quantitative approach to perceived health status: a validation study. $J$ Epidemiol Community Health 1980; 34: 281-6.

${ }^{2}$ Hunt S, McKenna S, McEwan J, Williams J, Papp E. Nottingham Health Profile: subjective health status and medical consultation. Soc Sci Med 1981; 15a: 221-9.

${ }^{3}$ Hunt S, McKenna S, Williams J. Reliability of a population survey tool for measuring perceived health problems: a study of patients with osteoarthritis. $J$ Epidemiol Community Health 1981; 35: 297-300.

‘ Hunt S, McEwan J, McKenna S. Measuring health status: a new tool for clinicians and epidemiologists. J Roy Coll Gen Practit 1985; 35: 185-8.

${ }^{5}$ McKenna S, Hunt S, McEwan J. Weighting the seriousness of perceived health problems using Thurstones method of paired comparisons. Internat $J$ Epidemiol 1981; 10: 93-7.

${ }^{6}$ Goldberg D, Hillier V. A scaled version of the General Health Questionnaire. Psychol Med 1979; 9: 139-45.

${ }^{7}$ Ebrahim S, Nouri F, Barer D. Measuring disability after a stroke. J Epidemiol Community Health 1985; 39: 86-9.

${ }^{8}$ Bridges K, Goldberg D. Psychiatric illness in inpatients with neurological disorders: patients' views on discussion of emotional problems with neurologists. $\mathrm{Br}$ Med J 1984; 289: 656-8.

${ }^{9}$ Folstein M, Maiberger R, McHugh P. Mood disorder as a specific complication of stroke. $J$ Neurol Neurosurg Psych 1977; 40: 1018-20.

${ }^{10}$ Robinson R, Starr L, Price T. A two year longitudinal study of mood disorders following stroke: prevalence and duration at six months follow up. Br J Psych 1984; 144: 256-62.

${ }^{11}$ Edwards J, Klemmack D. Correlates of life satisfaction: a re-examination. J Gerontol 1975; 28: 497-502.

${ }^{12}$ Neugarten B, Havighurst R, Tobin S. The measurement of life satisfaction. $J$ Gerontol 1961; 16: 134-43.

${ }^{13}$ Backett EM, McEwan J , Hunt S. Health and quality of life. Report to SSRC, London, 1981.

${ }^{14}$ Lipsey JR, Robinson RG, Pearlson GD, Rao K, Price TR. Nortriptyline treatment of post-stroke depression: a double blind study. Lancet 1984; i: 297-300. 